\title{
Myopic Changes in a Climber after Taking Acetazolamide and the Use of Corrective Lenses to Temporize Symptoms: A Case Report from Mount Kilimanjaro
}

\author{
Adam D. Hill, MD, FAWM \\ From the Department of Emergency Medicine, Icahn School of Medicine at Mount Sinai, New York, NY.
}

\begin{abstract}
When performing detailed tasks related to climbing or hiking, accurate vision is important for safety. Acetazolamide is a medication commonly used to prevent acute mountain sickness, but it has an uncommon side effect of transient myopia. Reports of this side effect are mainly associated with its use in obstetrics, where it is often prescribed in higher doses than used in acute mountain sickness prophylaxis. We describe the case of a climber taking low-dose acetazolamide who developed transient myopia. We further describe potential mechanisms of this rare side effect as well as a novel approach of field management utilizing possible materials at hand.
\end{abstract}

Key words: myopia, acetazolamide, altitude, altitude sickness, wilderness

\section{Introduction}

Acetazolamide (Diamox) is a carbonic anhydrase inhibitor with many clinical uses, including the prevention and treatment of acute mountain sickness (AMS). Dosing regimens vary greatly depending on the source one consults. The medication label ${ }^{1}$ advises a divided daily oral dose of $500 \mathrm{mg}$ to $1000 \mathrm{mg}$, while the Centers for Disease Control (CDC) Yellow Book and Wilderness Medical Society both suggest a lower twice-daily oral dose of $125 \mathrm{mg}$ for prevention and $250 \mathrm{mg}$ for treatment. ${ }^{2,3}$ Due to its use in high altitude travel, its potential side effects are important to consider in patient safety; these side effects include transient myopia. We describe a case of a climber on Mount Kilimanjaro who developed significant myopia after taking acetazolamide for AMS prevention as well as a novel approach to field management.

\section{Case Presentation}

A 35-year-old healthy woman with no significant medical history was part of a group of climbers attempting to summit Mount Kilimanjaro, Tanzania $(5895 \mathrm{~m})$. Of clinical

Corresponding author: Adam Hill, MD, 79-01 Broadway, Dept. of Emergency Medicine, B1-127, Queens, NY 11373 (e-mail: hilla@ nychhc.org).

Submitted for publication December 2015.

Accepted for publication April 2016. importance, she had a full eye examination by an ophthalmologist 8 months prior that was normal, with a selfreported baseline uncorrected visual acuity of 20/20 in the right eye (OD), 20/20 in the left eye (OS), and 20/20 when measured together $(\mathrm{OU})$. The day prior to starting the climb (Day 1), she began taking $125 \mathrm{mg}$ acetazolamide twice daily for prevention of AMS while staying in Arusha, Tanzania $(1400 \mathrm{~m})$, with the first dose being taken in the morning. In addition to acetazolamide, she was taking atovaquoneproguanil $250 \mathrm{mg} / 100 \mathrm{mg}$ daily for malaria prophylaxis and a norgestimate-ethinyl estradiol oral contraceptive. Within 12 hours of the first dose of acetazolamide, she began noticing slight changes in her ability to focus on objects at a distance. The following morning (Day 2), she noted a significant decrease in her visual acuity with severe blurring of distant objects.

At onset of the climb (Day 2), she was having difficulty managing the terrain due to the visual changes and in the late afternoon sought the advice of a physician who was part of the climbing party. An ocular field examination found normal pupil reactivity, accommodation, conjunctiva, extraocular motor function, and peripheral vision. Tonometry or other advanced ophthalmologic testing was not available to assess for elevated intraocular pressures or papilledema. There were no focal neurologic deficits. Aside from the visual changes, a full review of systems was positive only for increased urination and occasional paresthesias to hands 
and feet. A smartphone Snellen eye chart application (Eye Chart HD, Dok LLC) held at $1.25 \mathrm{~m}$ (4 feet, per application instructions) from the patient was used to determine a visual acuity of 20/70 OD, 20/70 OS, and $20 / 70$ OU. This information, combined with the otherwise grossly normal examination, painless nature of symptoms, absence of additional symptoms, the patient's lack of personal or familial ophthalmologic history, and the knowledge that the patient had been taking acetazolamide, led the physician to make a diagnosis of myopia. This diagnosis was further supported when the physician was able to secure a pair of corrective eyeglasses $(-2.5$ diopter) from a member of the climbing party. The eyeglasses returned the patient's vision to "nearly normal," per her report. This enabled her to continue the climb without significant impairment. The patient immediately discontinued acetazolamide and was started on $2 \mathrm{mg}$ dexamethasone every 6 hours for continued AMS prophylaxis. Prior to cessation of the acetazolamide on Day 2, only 4 doses had been administered.

The myopic climber received serial visual examinations by the physician during the remainder of the climb. The Table shows the trend of her visual acuity as determined by Snellen eye chart. Within 24 hours of acetazolamide cessation, she noted improvements in her vision and, although not yet at her baseline, she no longer required corrective lenses to safely negotiate the terrain. She successfully summited on Day 7, experiencing only mild AMS symptoms (headache and nausea) without recurrence of myopia or new visual disturbances. In-person follow-up 2 weeks later showed continued resolution of all symptoms and a stable visual acuity of 20/20 OD, 20/20 OS, and 20/20 OU when tested using a standard Snellen wall chart.

\section{Discussion}

Myopia, also called nearsightedness, is a condition in which images do not come into focus directly on the retina but instead in front of it. This results in reduced visual acuity of objects at a distance. A search of PubMed reveals that medical literature regarding transient myopia related to the use of acetazolamide is limited and consists mainly of case reports from the 1950s and 1960s. ${ }^{4-7}$ Although it is a documented side effect listed on the package insert ${ }^{1}$ as well as in popular medical references, such as UpToDate (UpToDate, Inc.) and Epocrates (athenahealth, Inc.), it is not one that is commonly discussed among medical experts providing care or advice to individuals utilizing the drug for prevention or treatment of AMS. There has been a case report of a trekker in Nepal experiencing visual changes while taking acetazolamide for the treatment of AMS at doses of $250 \mathrm{mg}$, but in that situation the dosage of acetazolamide was higher than described here; myopia began after the patient was at high altitude and already experiencing AMS. In that case, serial visual examinations and attempted correction with lenses did not occur. ${ }^{8}$

Acetazolamide is a carbonic anhydrase inhibitor within the sulfonamide drug class. It was initially used for its diuretic and gastric acid suppression properties. In the 1960s, its potential for affecting physiology at altitude became recognized and studied. ${ }^{7}$ It has been recommended as first line prophylaxis by the Wilderness Medical Society for prevention of AMS in doses of 125 mg orally twice a day. ${ }^{2}$ Its ability to alter the body's response to altitude is not fully understood, but is believed to result from a combination of metabolic acidosis through increased bicarbonate excretion, increased minute ventilation, and blunted hypoxic pulmonary hypertension. ${ }^{9}$ A few of the more commonly noted side effects are paresthesias, taste alterations, and urinary frequency. ${ }^{1}$

Transient myopia has been previously described in multiple sulfonamide medications and is believed to be due to edema of the ciliary body, but the exact mechanism of the myopia is unknown. ${ }^{10-12}$ The

Table. Visual acuity in relation to day of climb and time after acetazolamide cessation

\begin{tabular}{ccccc}
\hline Day & Right eye $($ OD $)$ & Left eye $($ OS $)$ & Together $($ OU $)$ & Hours post-cessation \\
\hline \multirow{2}{*}{$20 / 70$} & $20 / 70$ & $20 / 70$ & 0 \\
3 & $20 / 25$ & $20 / 40$ & $20 / 25$ & 24 \\
4 & $20 / 25$ & $20 / 30$ & $20 / 25$ & 48 \\
5 & $20 / 20$ & $20 / 25$ & $20 / 20$ & 72 \\
6 & $20 / 20$ & $20 / 25$ & $20 / 20$ & 96 \\
8 & $20 / 20$ & $20 / 20$ & $20 / 20$ & 144 \\
$14^{*}$ & $20 / 20$ & $20 / 20$ & $20 / 20$ & - \\
\hline
\end{tabular}

Readings obtained using Eye Chart HD application without corrective lenses.

* Indicates reading obtained using standard Snellen wall chart. 
dominant theory is that edema of the ciliary body occurs, resulting in curvature of the lens and possible narrowing of the anterior chamber. ${ }^{6,10-12}$ It is unclear if this edema is due to a sensitivity reaction or to a medication-induced influx of prostaglandins at the level of the ciliary body. One of the original theories regarding the induced myopia proposed that it was due to a reduction in aqueous humor production in the anterior chamber secondary to acetazolamide's inhibition of carbonic anhydrase. ${ }^{5}$ Without a subsequent increase in outflow resistance to keep the volume constant in the anterior chamber, a forward shift of the iris-lens diaphragm would occur. ${ }^{5}$ However, this theory did not account for the fact that many sulfonamide medications lack carbonic anhydrase inhibition yet still result in a similar myopic event or for the measured increases in ciliary body thickness. This suggests that its myopic effect may be due to its sulfonamide structure rather than its inhibitory effect on carbonic anhydrase. It should also be noted that a multitude of other medications have been associated with transient myopia, including several that are historically or commonly used in travel and high-altitude environments, such as promethazine hydrochloride (Phenergan), prochlorperazine (Compazine), metronidazole (Flagyl), and aspirin, although different mechanisms are at play. ${ }^{13}$

The occurrence rate of this myopia is unknown. Typically, the patient will have anywhere from 1 to 8 diopters of myopia. ${ }^{14}$ Myopia can start as quickly as 4 hours after administration or have a delayed onset of 5 days. ${ }^{14}$ It is not currently possible to predict who will develop this myopia, and it has been noted that repeat exposure does not predictably cause recurrence. ${ }^{12}$ Thankfully, this effect is transient and, in all reported cases, has completely resolved within several days of cessation of the medication. ${ }^{12,14}$

Due to acetazolamide's frequency of use for prevention and treatment of AMS, it is advisable to discuss this side effect with individuals planning to travel to high altitude regions. Because many of these users will be climbing or performing technical skills in those environments, a decrease in visual acuity puts them at a marked disadvantage and risk for injury. This injury risk could occur in a number of scenarios, including an inability to navigate obstacles while walking/climbing or improper rigging/usage of safety gear. It is therefore prudent to advise climbers planning to use acetazolamide to either take a brief course of the medication far in advance of departure or to initiate the medication several days before any hiking or technical skills are required on the trip itself. ${ }^{15}$ A recommendation of prompt cessation upon development of visual changes is warranted. Additionally, the utilization of other medications for AMS prophylaxis and treatment, such as dexamethasone, should be considered. Although this myopia is innocuous in the sense that it is transient and ultimately resolves without intervention other than medication cessation, the increased risk of injury needs to be discussed.

The health care provider in this scenario was wise to utilize the corrective lenses at hand to aid the patient. By recognizing the potential for something as common as a pair of corrective eyeglasses to counter the myopic effects, a simple temporizing solution was found. Being aware that myopia is the most common refractive error in the general population made it more likely that any eyeglasses obtained would be of potential benefit to the patient. This was accomplished by recognizing this side effect and then taking stock of available gear within the climbing party. By doing so, potential injury was minimized and trip cancellation avoided while the symptoms resolved.

Acknowledgments: The author acknowledges John Mose Roberts, Jr., MD of the Colorado Eye Institute, Colorado Springs, CO, for technical help.

Author Contributions: Study concept and design (ADH); obtaining funding (N/A); acquisition of the data (ADH); analysis of the data $(\mathrm{ADH})$; drafting of the manuscript $(\mathrm{ADH})$; critical revision of the manuscript (ADH); approval of final manuscript (ADH).

Financial/Material Support: None.

Disclosures: None.

\section{References}

1. Acetazolamide Drug Label (2010). Available at: http:// dailymed.nlm.nih.gov/dailymed/drugInfo.cfm?setid=c1c7 6f21-e1fc-40ea-947a-bc9c752f80c8. Accessed November $8,2015$.

2. Luks AM, McIntosh SE, Grissom CK, et al. Wilderness Medical Society practice guidelines for the prevention and treatment of acute altitude illness: 2014 update. Wilderness Environ Med. 2014;25:S4-S14.

3. Hackett PH, Shlim DR. Altitude illness. In: Brunette GW, ed. CDC Health Information for International Travel 2016. New York, NY: Oxford University Press; 2016: 68-74.

4. Binder SS, Steele W. Acute transient myopia associated with use of acetazolamide (diamox). J Am Med Assoc. 1957; 165:154-155.

5. Muirhead JF, Scheie HG. Transient myopia after acetazolamide. Arch Ophthalmol. 1960;63:315-318.

6. Galin MA, Baras I, Zweifach P. Diamox-induced myopia. Am J Ophthalmol. 1962;54:237-240.

7. Swenson ER. Carbonic anhydrase inhibitors and high altitude illness. Subcell Biochem. 2014;75:361-386.

8. Szawarski P, Hall-Thompson B. Acetazolamide-induced myopia at altitude. Wilderness Environ Med. 2009;20: 300-301.

9. Hackett PH, Roach RC. High altitude medicine and physiology. In: Auerbach PS, ed. Wilderness Medicine. 6th ed. Philadelphia, PA: Mosby; 2012:2-33. 
10. Araujo JR, Silva SE, Cruz F, Falcao-Reis FF. Acute transient myopia with shallowing of the anterior chamber induced by sulfamethoxazole in a patient with pseudoxanthoma elasticum. J Glaucoma. 2014;23:415-417.

11. Bovino JA, Marcus DF. The mechanism of transient myopia induced by sulfonamide therapy. Am J Ophthalmol. 1982;94:99-102.

12. Krieg PH, Schipper I. Drug-induced ciliary body oedema: a new theory. Eye (Lond) 1996;10:121-126.
13. Kaimbo DKW. Transient drug-induced myopia. SAJ Case Rep. 2014;1:105.

14. Grant WM, Schuman JS. Acetazolamide. In Toxicology of the Eye: Effects on the Eyes and Visual System from Chemicals, Drugs, Metals and Minerals, Plants, Toxins and Venoms; also Systemic Side Effects from Eye Medications. 4th ed. Springfield, IL: Charles C. Thomas; 1993.

15. Bärtsch P, Swenson ER. Acute high-altitude illnesses. N Engl J Med. 2013;368:2294-2302. 